\title{
Re-Irradiation with Conformal Electron Beam Therapy for Recurrent Breast Cancer
}

\author{
Ariel E Pollock ${ }^{1}$, Ernest Butler ${ }^{2}$ and Jondavid Pollock ${ }^{2 *}$ \\ ${ }^{1}$ Department of Radiation Oncology, The University of Maryland School of Medicine, US \\ ${ }^{2}$ Division of Radiation Oncology, The Schiffler Cancer Center, Wheeling Hospital, US
}

Submission: October 22, 2019; Published: October 30, 2019

*Corresponding author: Jondavid Pollock, Division of Radiation Oncology, The Schiffler Cancer Center, Wheeling Hospital, Wheeling, WV 26003, West Virginia, US

Abstract

Patients with recurrent breast cancer involving the chest wall, who have been previously irradiated, present a clinical challenge. Pulsed reduced dose rate external beam radiotherapy has been shown to reduce recurrences with an acceptable rate of toxicity including chest wall soft tissue and rib necrosis [1]. For patients presenting with a breast cancer recurrence in close proximity to the skin surface, our treatment center has modified the technique by using electrons passing through customized wax bolus milled by a commercial compensator manufacturer (decimal, Sanford, FL).

Keywords: Breast cancer; Radiotherapy; Photon; Electron beam therapy; CT scan; Clinical tumor; Oligore current disease; Compensator

\section{Introduction}

Three breast cancer patients with locally recurrent disease on a previously irradiated chest wall presented for evaluation. All had received previous chest wall radiotherapy to a dose of 5040cGy using high energy tangentially-directed photons, followed by as much as a $1600 \mathrm{cGy}$ electron beam boost to the mastectomy scar or tumor bed $(4 \mathrm{MeV}$ electrons were utilized dosed to the $90 \%$ isoodose line with a $1 \mathrm{~cm}$ bolus; effective depth of penetration was $1 \mathrm{~cm}$ ). At a median disease-free interval of 18 months (range 12-30 months), patients presented with oligorecurrent disease involving the chest wall only. Staging labs and radiographs indicated no evidence of hematogenous metastases. All patients underwent a gross total resection of all visible disease prior to receiving radiotherapy, and pathological margins were reported as no tumor on ink.

\section{Materials and Methods}

The patients were treated using electron compensators with a reduced dose-rate technique. The electron compensators were designed to precisely match the patient's CT based surface contour. The compensators were milled from hard wax based on a computer-generated specification which incorporated gantry angle, tumor size, position on the chest wall, and depth required for tumor coverage. The wax density was $0.92 \mathrm{~g} / \mathrm{cc}$ and heterogeneity calculations were applied to the treatment planning. Treatments were delivered using $10-12 \mathrm{MeV}$ electrons prescribed such that the $90 \%$ isodose line covered the deepest extent of the clinical tumor volume. Thirty-eight fractions of $120 \mathrm{cGy}$ were delivered twice daily with a 6 hour inter-treatment interval for a total dose of $4560 \mathrm{cGy}$. An effective dose rate of $5 \mathrm{cGy} /$ minute was achieved by breaking up each treatment into 10 sub-fractions with a delay between each sub-fraction; the linear accelerator used could generate a dose rate of no less than $400 \mathrm{cGy} /$ minute. Each sub-fraction delivered 12cGy with a delay of 2 minutes and 24 seconds between beam-on times over the course of 24 minutes.

\section{Results}

Table 1 illustrates the details regarding each of the three patients treated in this manner. Patients were treated over the course of 4 weeks without interruption. With a median follow up of 24 months (range 24-29 months), there have been no observed recurrences in the treatment field. Skin toxicity was significantly less that would be expected based on our prior experience, as well as that in the literature, with conventional standard dose and dose-rate radiotherapy [2,3]. One patient developed fibrosis of the axillary fold and one had slight erythema. No moist desquamation, skin or chest wall necrosis was observed. 
Table 1: Summary of patients receiving reduced dose rate compensated electron beam therapy.

\begin{tabular}{|c|c|c|c|c|}
\hline Pt & Dx & Prior RT & Conformal e Beam & Notes \\
\hline \multirow{2}{*}{ PA } & 2000; bilat mst & 2000; 50Gy L cw, sc, axilla & 4560cGy 10MeV BID & 29 mos disease free \\
\cline { 2 - 5 } & 2009; T4, ER/Her +ve & 2009; 45Gy L sc & & fibrosis without pain \\
\hline \multirow{2}{*}{ CW } & 1997; TxN1 lump & 1998; 5040cGy breast, axilla & 4560cGy 10MeV BID & 24 mos disease free \\
\cline { 2 - 5 } & $2009 ;$ R mst for recur & & 1600cGy scar boost & minimal erythema only \\
\hline \multirow{2}{*}{ PM } & 2006; T1N1 lump & 2006; 5040cGy breast & 4560cGy 12MeV BID & 24 mos disease free \\
\cline { 2 - 5 } & 2009; mst for cw recur & 1400cGy scar boost & & no acute toxicity \\
\hline
\end{tabular}

\section{Conclusion}

This case study of three patients demonstrates that reduced dose rate electron beam therapy, using customized wax-based compensators, results in excellent local control and tolerable morbidity in patients receiving as much as $112 \mathrm{~Gy}$ for an isolated chest wall recurrence of breast cancer.

\section{References}

1. Richards GM, Tome WA, Robins HI, Stewart JA, Welsh JS, et al. (2009) Pulsed reduced dose-rate radiotherapy: a novel locoregional retreat- ment strategy for breast cancer recurrence in the previously irradiated chest wall, axilla, or supraclavicular region. Breast Cancer Res Treat 114(2): 307-313.

2. Wahl AO, Rademaker A, Kiel KD, Marks LB, Croog V, et al. (2008) Multi-institutional review of repeat irradiation of chest wall and breast for recurrent cancer Int J Radiat Oncol Biol Phys 70(2): 477-484.

3. Oldenborg S, Valk C, van Os R, Venselaar J, Vörding PZ, et al. (2016) Rib fractures after reirradiation plus hyperthermia for recurrent breast cancer: predictive factors. Strahlenther Onkol 192(4): 240-247. 